\title{
Myofibroblastäre Sarkome der Brust - extrem rar und ob der schlechten Datenlage diffizil zu therapieren
}

\section{Einleitung}

Brustsarkome sind selten, sie machen weniger als $1 \%$ der maligen Brusttumoren aus und sind histologisch heterogen. Sie entstehen entweder de novo, im Rahmen eines chronischen Lymphstaus oder nach einer Radiotherapie, wobei es sich bei den letzten 2 Möglichkeiten vor allem um Angiosarkome handelt. Das mittlere Erkrankungsalter liegt bei 45 - 50 Jahren, wobei zu $99 \%$ Frauen betroffen sind.

Es existiert angesichts der Seltenheit und der erst in den letzten Jahren verfügbaren spezifischen immunhistochemischen Antikörper, durch die die Diagnose vereinfacht und präzisiert wird, keine einheitliche histologische Unterteilung der Brustsarkome.

Die mammografische Diagnose eines Brustsarkoms ist schwierig, da es keine eindeutigen pathognomonischen radiologischen Veränderungen gibt; so präsentiert es sich meist als nichtkalzifizierender, hypervaskularisierter, ovaloider und nicht

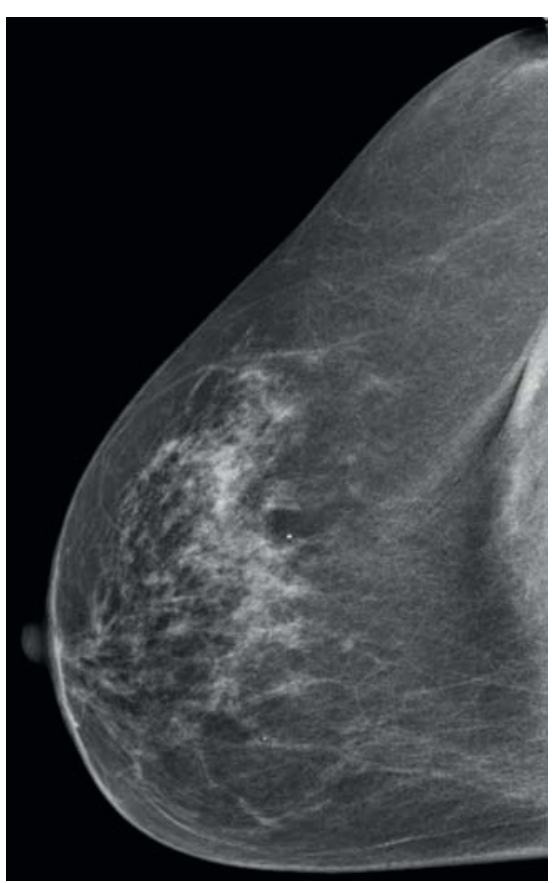

- Abb. 1 Konventionelle Mammografie des Brusttumors: Das Brustdrüsengewebe wird beinahe gänzlich von einer homogen hyperdensen Expansion eingenommen. klar begrenzter großer Tumor - die Biopsie zur genauen Diagnosestellung ist somit wie üblich unabdingbar. Ergänzend ist eine MR-Mammografie zur exakteren Größenausdehnungsbestimmung hilfreich. Da Brustsarkome vor allem in die Lungen metastasieren, sollte ein CT des Thorax zum Staging durchgeführt werden. Ein Abdomen-Becken-CT und ein Wirbelsäulen-MRT sind aufgrund der Metastasierungstendenz bei rundzelligen/myxoiden Liposarkomen zu ergänzen, ebenso der Ausschluss von Knochen- und zentralnervösen Sekundärabsiedelungen bei Angiosarkomen.

Therapeutisch spielt bei Brustsarkomen die chirurgische R0-Resektion mit entsprechend großem Sicherheitsabstand (1$2 \mathrm{~cm}$ ) die prognostisch wichtigste Rolle. Die neoadjuvante beziehungsweise adjuvante Strahlen- und Chemotherapie ist speziellen Situationen vorbehalten. Anhand dieses Fallberichts wird vor allem das Therapiemanagement erläutert.

\section{Kasuistik}

Wir berichten über eine 57-jährige postmenopausale Patientin mit unauffälliger Familienanamnese bezüglich Mammaund Ovarialkarzinom, die aufgrund eines rasch wachsenden Tumors der linken Brust unser Brustgesundheitszentrum aufsuchte ( $\triangleright$ Abb. 1). Der Tumor präsentierte sich klinisch als kleinkindskopfgroß, mit

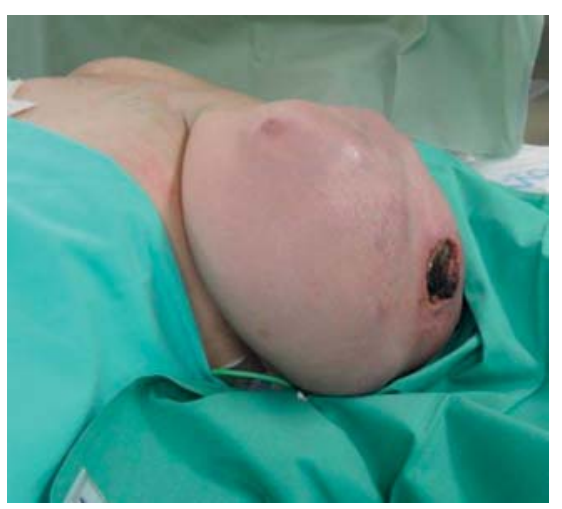

- Abb. 2 Intraoperative Ansicht: kleinkindskopfgroßer, livide verfärbter, lokal exulzerierter Tumor bei 3 Uhr. livide verfärbter gespannter Haut und lokal bei 3 Uhr exulzeriert ( $\triangleright$ Abb. 2). Die Stanzbiopsie war nicht aussagekräftig; es bestand der Verdacht auf einen mesenchymalen Tumor. Die Staging-Untersuchungen mit einer Sonografie der Axilla, einem CT des Thorax und des Abdomens/ Beckens sowie einer Ganzkörper-Skelettszintigrafie waren unauffällig, die Tumormarker CEA und CA 15 - 3 im Normbereich. Nach Diskusssion im interdisziplinären Tumorboard wurde die Patientin bei Möglichkeit zur R0-Resektion primär mit einer Mastektomie unter Mitnahme der Pectoralisfascie und Sentinellymphknotenentfernung operativ versorgt. Die endgültige Histologie ergab ein Sarkom vom myofibroblastären Typ G3 N0 (sn) mit einem minimalen Resektionsabstand nach pektoral von $4 \mathrm{~mm}$ ( $\triangleright$ Abb. 3). Adjuvant erhielt die Patientin sequenziell eine Chemotherapie mit 4 Zyklen (3-wöchentlich) Epirubicin und Ifosfamid (Tag 1 und 2: jeweils $50 \mathrm{mg} / \mathrm{m}^{2}$ Epirubicin - entspricht $100 \%$-Dosis, über 30 min; Tag 1 bis 5: $1800 \mathrm{mg} / \mathrm{m}^{2}$ Ifosfamid - entspricht $100 \%$-Dosis, über $1 \mathrm{~h}$ ) und anschließend eine Bestrahlung der Thoraxwand mit einer Gesamtdosis von 50 Gy (Einzeldosis 2 Gy) sowie folgend einer Aufsättigung der Ablationsnarbe mit insgesamt $10 \mathrm{~Gy}$ (Einzeldosis 2 Gy). Seit 20 Monaten ist die Patientin nunmehr rezidivfrei bei uneingeschränkter körperlicher Belastbarkeit (ECOG 0).

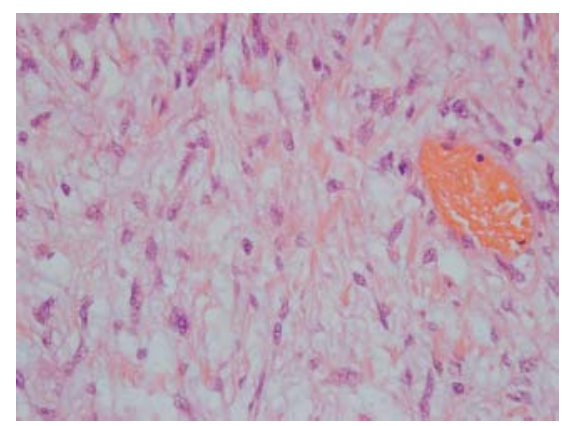

- Abb. 3 Histologie-Ausschnitt des myofibroblastären Mammasarkoms: uniforme, meist spindelige Zellelemente, in kurzen, einander durchflechtenden Bündeln gelagert. 


\section{Diskussion}

Das in diesem Fall vorgefundene myofibroblastäre Sarkom (syn. Myofibrosarkom, Sarkom mit myofibroblastischer Differenzierung) ist eine Rarität; es existieren nur wenige Fallberichte. Leitliniengerechte Behandlungsstrategien fehlen daher. Wir haben unsere Therapie und Nachsorge an die anderer Brustsarkome angelehnt. Sie sollten primär wie R0-resektable Tumoren operativ versorgt werden, wobei ein Sicherheitsabstand von $1-2 \mathrm{~cm}$ anzustreben ist, woraus sich auch die Operationstechnik (Lumpektomie/Ablatio mammae) ergibt. Die primäre generelle Mastektomie ist der Tumorektomie bezüglich der Lokalrezidivrate nicht überlegen. Ob eine Sentinellymphknotenentfernung sinnvoll ist, kann nicht beantwortet werden; wir haben sie nur deshalb durchgeführt, weil die primäre Stanzbiopsie des Brusttumors keine definitive histologische Diagnose erbrachte und vor allem metaplastische Karzinome und Karzinosarkome eine deutlich erhöhte Inzidenz an axillären Lymphknotenmetastasen zeigen. Zur adjuvanten Maximaltherapie (Strahlen- und Chemotherapie) haben wir uns aufgrund der schlechten Tumordifferenzierung (G3), der Tumorgröße $(>5 \mathrm{~cm}$ ) und des knappen pektoralen Resektionsabstands (4 mm) entschieden. Bei der Nachsorge orientieren wir uns an den Richtlinien der Sarkomnachsorge der Rumpfsarkome.

\section{FAZIT}

Subsumierend ist die Therapie des myofibroblastären Sarkoms der Brust angesichts der schlechten Datenlage auf jede/n Patienten/in individuell in Zusammenarbeit mit Radiologen, Pathologen, Onkologen, Chirurgen und Strahlentherapeuten maßzuschneidern, das Anlehnen der Behandlung an die der „allgemeinen“ Brustsarkome hat sich in unserem Fall bewährt. Studien sind wegen der geringen Fallzahlen schwierig durchzuführen, lediglich anhand von Fallberichten gelingt es, einen Einblick in die Thematik zu erhalten - was auch der Anstoß war, diesen Artikel zu verfassen.

\section{Interessenkonflikt}

Die Autoren erklären, dass keine Interessenkonflikte bestehen.
Autoren

M. Themel' , S. Sauseng', E. Mathew' ${ }^{1}$, T. Niernberger ${ }^{1}$, S. Gabor ${ }^{1}$, W. Herz' ${ }^{1}$, R. Stering ${ }^{2}$, H. Rabl ${ }^{1}$

\section{${ }^{1}$ Abteilung für Allgemeinchirurgie - Brustgesundheitszentrum Landesklinikum Hochsteiermark Standort Leoben \\ ${ }^{2}$ Abteilung für Pathologie Landesklinikum Hochsteiermark Standort Leoben}

\section{Korrespondenzadresse}

Dr. Michael Themel

LKH Hochsteiermark Standort Leoben

Vordernberger Straße 42

A-8700 Leoben

MichaelJuergen.Themel@kages.at

Literatur

Literatur beim Verfasser.

Bibliografie

DOI http://dx.doi.org/10.1055/s-0042-111681 | Senologie 2017; 14: 14-15

(c) Georg Thieme Verlag KG Stuttgart · New York ISSN 1611-6453 